\title{
Aneurysmal sac located between right pulmonary artery and left atrium
}

\author{
Aysenur Pac, MD, ${ }^{a}$ Ugursay Kiziltepe, MD, ${ }^{b}$ Ozcan Ozeke, MD, ${ }^{\mathrm{c}}$ Zehra Golbasi, MD, ${ }^{\mathrm{a}}$ and Mustafa Pac, MD, \\ Ankara, Turkey
}

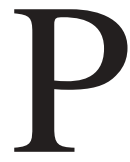

ulmonary arteriovenous malformations (PAVMs) are abnormal connections between pulmonary arteries (PAs) and pulmonary veins (PVs). ${ }^{1}$ These lesions may cause cyanosis, clubbing, and dyspnea on exertion and can be misdiagnosed as complex congenital heart disease. We report an interesting case with a large aneurysmal sac in continuity with the left atrium (LA) and fed by a large PA branch.

\section{Clinical Summary}

A 6-year-old boy had dyspnea and severe cyanosis, which first appeared at 6 months of age, and was admitted to our hospital. Mucocutaneous telengiectasias were not present. A negative pathergy test ruled out Behcet's disease. His echocardiography revealed an aneurysmal sac behind the LA, which was in continuity with the LA via a large orifice and absent adjunctive defects. Angiography demonstrated a wide connection between the sac and LA and a large, single feeding artery (afferent) originating from the right PA without an efferent vessel from the sac (Figure 1,a). Cardiac 16-slice computerized tomographic angiography showed that PV orifices were not in relation with the sac and no additional PAVMs were present. All the diagnostic tests supported the absence of thrombi or calcium.

A right thoracotomy through the fourth intercostal space was performed, and an aneurysm was found posteroinferior of the right lower lobe. It was not covered with parenchyma and was adhered loosely to surrounding lung tissue. The feeding artery was found at superomedioposterior aspect of aneurysm and ligated and divided (Figure 1,b). A Satinsky clamp was placed over the transition line and the aneurysm was opened. There were no intraluminal thrombi. Following resection of the aneurysm wall, the LA was oversewn. The patient's postoperative course was uneventful. Pathologic examination of the sac wall revealed aneurysm tissue originated from vessel wall. The patient was discharged from the hospital on postoperative day 6 with an arterial saturation of $97 \%$ in room air. At postoperative month 6 , his functional capacity was in New York Heart Association class I and he was still fully saturated.

From the Departments of Pediatric Cardiology, ${ }^{a}$ Cardiovascular Surgery, ${ }^{\mathrm{b}}$ and Cardiology, ${ }^{\mathrm{c}}$ Turkiye Yuksek Ihtisas Hospital, Ankara, Turkey.

Presented in the 4th World Congress of Pediatric Cardiology and Cardiothoracic Surgery, September 18-23, 2005, Buenos Aires, Argentina.

Received for publication Oct 10, 2005; accepted for publication Oct 19, 2005 .

Address for reprints: Dr Ugursay Kiziltepe, 30. Cad. 21/23, Isci Bloklari Mah, Karakusunlar, Balgat, Ankara 06000 Turkey (E-mail: ukiziltepe@ttnet. net.tr).

J Thorac Cardiovasc Surg 2006;131:1187-8

$0022-5223 / \$ 32.00$

Copyright (C) 2006 by The American Association for Thoracic Surgery doi:10.1016/j.jtcvs.2005.10.059
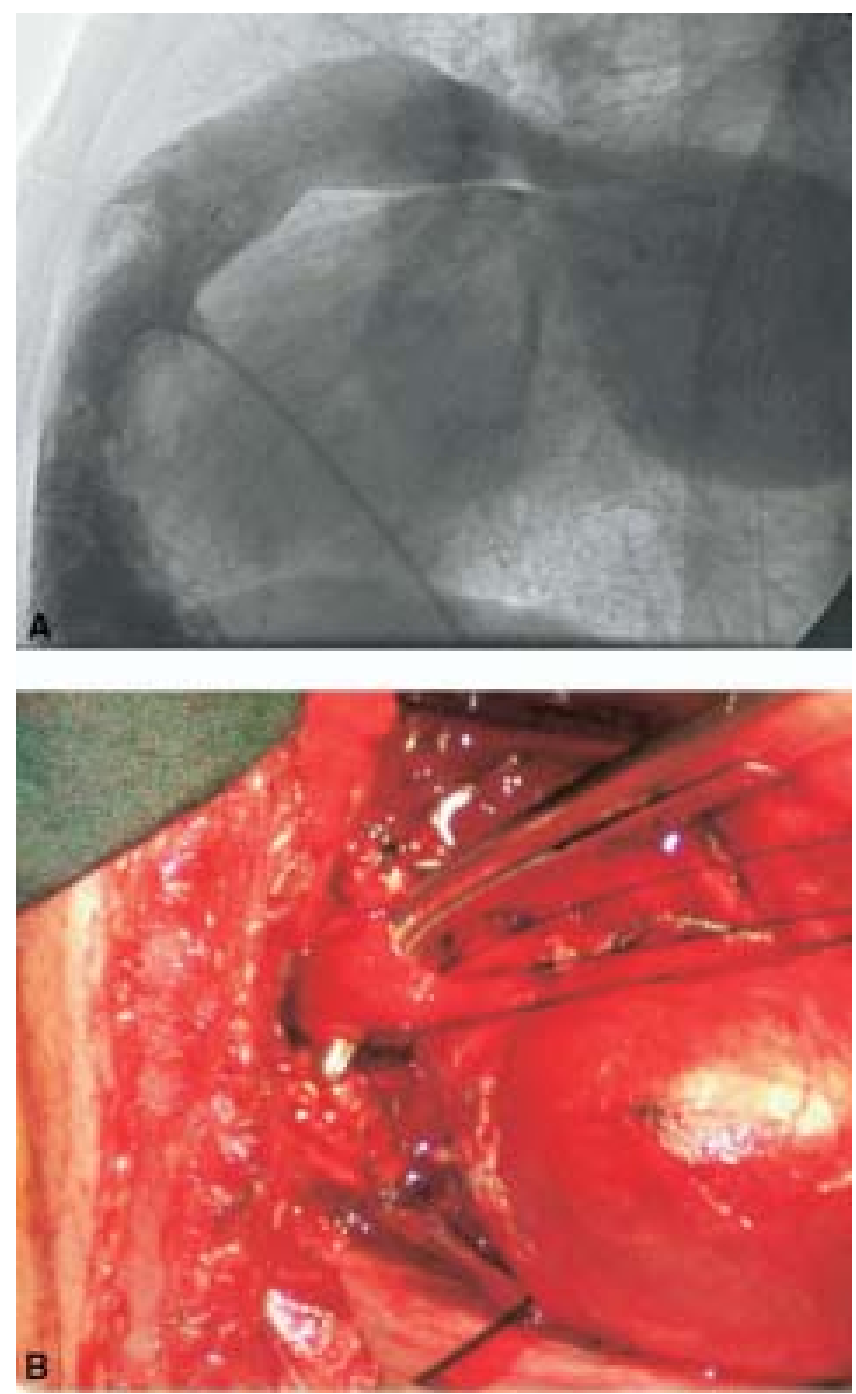

Figure 1. A, Angiographic appearance. Proximity of afferent artery and LA-aneurysm connection are noticeable (lateral view). B, Surgical view. Extraparenchymally located, thin-walled aneurysmal sac with afferent artery originating from the right PA.

\section{Discussion}

PAVMs are most commonly congenital in nature ${ }^{1}$ and $47 \%$ to $70 \%$ of the cases are associated with hereditary hemorrhagic telangiectasia. $^{2}$ Development of PAVMs is a well-known phenomenon following cavopulmonary anastomoses, especially in the absence of hepatic blood flow to the lungs. ${ }^{3}$ All PAVMs have afferent supply, usually from branches of the PA. The efferent limb of an 
arteriovenous malformation drains into branches of PVs or sometimes directly into the LA or inferior vena cava. They usually are embedded to lung parenchyma.

In addition to causing shunt resulting in dyspnea, volume overload, and cyanosis, PAVMs also cause the loss of filter function of lungs and thus may allow development of stroke and cerebral abscess. The classic triad of dyspnea, cyanosis, and clubbing is present in only $10 \%$ of cases. ${ }^{1}$

In our case, the lesion was producing high-output right-to-left shunt and causing severe cyanosis. The aneurysm had a single large feeding artery (afferent vessel) and no efferent vessel. Interestingly, it was in continuity with the LA with an orifice as big as the aneurysm itself. In our knowledge no similar case has been reported in literature.

Traditional options for the definitive management of PAVM include embolization and surgery. Ligation, local excision, segmentectomy, lobectomy, or pneumonectomy have been performed, ${ }^{1,2}$ although most of the time parenchyme-saving operations are preferred.

It was obvious that embolization would be inappropriate for this case not only because leaving a residual aneurysm would result in a high risk of rupture but also because of the unsuitable anatomy of the feeding artery. During preoperative evaluation we considered 2 possible approaches for surgical treatment of this PAVM. Instead of median sternotomy and repair through the LA with cardiopulmonary bypass and cardiac arrest, we used simple ligation and resection through thoracotomy, which allowed better exposure, a shorter operation, and faster recovery. We believe the origin of this sac would be one of the following: an aneurysmal PAVM with draining vessel was absorbed in time and united widely to the LA, or a distal PA aneurysm ruptured and united to the LA. Unusual features like extraparenchymal location of the lesion, absence of an efferent vessel, unity of aneurysmal sac with the LA, and the absence of associated etiologic factors make this lesion interesting. Nonetheless, prompt surgical treatment with a simple approach relieved the severe symptoms and prevented a potential fatal rupture.

In conclusion, although PAVMs are rare, they should be included in any differential diagnosis of worsening dyspnea, cyanosis, stroke, or hemoptysis. Their presence should be considered especially in patients with cyanosis whom no evidence of intracardiac shunts in echocardiography. Prompt workup and intervention can prevent fatal complications like rupture and lead to dramatic improvement in symptoms.

\section{References}

1. Gossage JR, Kanj G. Pulmonary arteriovenous malformations. A state of the art review. Am J Respir Crit Care Med. 1998;158:643-61.

2. Khurshid I, Downie Gh. Pulmonary arteriovenous malformation. Postgrad Med J. 2002;78:191-7.

3. Duncan BW, Desai S. Pulmonary arteriovenous malformations after cavopulmonary anastomosis. Ann Thorac Surg. 2003;76:1759-66.

\title{
Use of the endoclamp device in a patient with a native mitral valve endocarditis and a previous arch replacement with a modified Griepp technique
}

\author{
Siamak Mohammadi, MD, Pierre Voisine, MD, Patrick Mathieu, MD, and François Dagenais, MD, Québec City, Canada
}

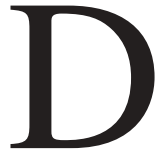

evelopment of new catheters and cannulas has allowed accessing the heart through short incisions. Use of this technology may also be applied for difficult reoperative situations. We herein report the use of the endoclamp in a patient with a previous root and arch replacement requiring mitral surgery for a native mitral endocarditis.

\footnotetext{
From the Department of Cardiac Surgery, Laval Hospital, Québec City, Québec Canada.

Received for publication Nov 25, 2005; accepted for publication Jan 3, 2006.

Address for reprints: Dr François Dagenais, Department of Cardiac Surgery, Laval Hospital, 2725 Chemin Ste-Foy, Québec, Canada, G1V 4G5

(E-mail: francois.dagenais@chg.ulaval.ca).

J Thorac Cardiovasc Surg 2006;131:1188-90

$0022-5223 / \$ 32.00$

Copyright $\odot 2006$ by The American Association for Thoracic Surgery doi:10.1016/j.jtcvs.2006.01.007
}

\section{Clinical Summary}

A 58-year-old male was admitted with asthenia, weight loss, and fever. His past medical history was positive for hypertension, diabetes, peripheral vascular disease (aortobifemoral graft). The patient had a root replacement with a Bjork-Shiley conduit in 1982 for a type A dissection. The patient also had an arch replacement using an isolated graft to the arch vessels (modified Greipp technique) and an "elephant trunk" procedure in 2001 with a right axillary artery cannulation. The patient also required a replacement of his thoracoabdominal aorta in May 2002.

Transthoracic echocardiography revealed a moderate mitral regurgitation and a $3.5-\mathrm{cm}$ vegetation on the posterior leaflet. No vegetation was documented on the Bjork-Shiley aortic prosthesis. Blood cultures grew Staphylococcus epidermidis. Owing to his poor overall condition, the patient was treated with vancomycin and rifampycin although the embolic risk was present. Absence of dacron graft infection was suggested by a normal gallium scan and magnetic resonance imaging. 